\title{
Improving the STEM Spatial Resolution Limit
}

Ondrej L. Krivanek ${ }^{1,2}$, Andrew L. Bleloch ${ }^{1}$, Niklas Dellby ${ }^{1}$, Tracy C. Lovejoy ${ }^{1}$, Chenglong Shi ${ }^{1,3}$ and Wu Zhou ${ }^{4}$

1. Nion R\&D, Kirkland, WA.

2. Department of Physics, Arizona State University, Tempe, AZ.

3. School of Physics, Peking University, Beijing, China.

4. Laboratory of Vacuum Physics, University of Chinese Academy of Sciences, Beijing, China.

Professor Hashimoto pioneered several new electron microscope capabilities and applied them to many key problems. His dedication to making major contributions in every field he entered was a great inspiration, and it is very appropriate to dedicate this short paper on STEM resolution in his memory.

Scanning transmission electron microscopy (STEM) has progressed very significantly in recent years. Improving the spatial resolution by aberration correction has led to $1 \AA(100 \mathrm{pm})$ spatial resolution becoming routine at primary energies as low as $60 \mathrm{keV}$ in cold field emission (CFEG) STEM [1-3], and probe currents of $\sim 1 \mathrm{nA}$ have become available in sub- $2 \AA$ probes at higher keVs. Another important development was the introduction of ultra-high energy resolution monochromators and spectrometers, which have made $5 \mathrm{meV}$ energy resolution electron energy loss spectroscopy (EELS) possible [4].

The quest for improved spatial resolution over the last two decades is illustrated in Fig. 1. The resolution and the probe size are closely related, and we prefer to focus on the probe size, which can be defined without needing a test object with suitable spacings. The probe size depends on the electron wavelength $\lambda$ and therefore the primary energy and also on the source size contribution, which is proportional to $\left(\mathrm{I}_{\mathrm{p}} / \mathrm{I}_{\mathrm{c}}\right)^{0.5}$, where $\mathrm{I}_{\mathrm{p}}$ is the probe current and $\mathrm{I}_{\mathrm{c}}$ the coherent current (=current in a probe that's $50 \%$ coherent), and the contribution is added in quadrature to the diffraction-limited probe size [3]. The orange curves in Fig. 1 show the probe size for uncorrected STEM computed using formulas described in [3], for $\mathrm{I}_{\mathrm{p}} / \mathrm{I}_{\mathrm{c}}=0.1$ (solid curve) and $\mathrm{I}_{\mathrm{p}} / \mathrm{I}_{\mathrm{c}}=0.5$ (dashed curve), i.e. for about 15 and $75 \mathrm{pA}$ probe current in the CFEG case. They show that for $\mathrm{C}_{\mathrm{s}}=0.5 \mathrm{~mm}$, probe size of $135 \mathrm{pm}$ was attainable at $200 \mathrm{keV}$ in uncorrected STEM, with a severely limited probe current. At $100 \mathrm{keV}$ with $\mathrm{C}_{\mathrm{s}}=0.7 \mathrm{~mm}$ (VG STEM with ultra-high resolution OL polepiece), the minimum probe size was about $200 \mathrm{pm}$.

STEM aberration correctors initially improved this performance about $2 \mathrm{x}$, with $100 \mathrm{pm}$ probe sizes becoming possible at $100-120 \mathrm{keV}$ [1], and $<78 \mathrm{pm}$ probe sizes at $300 \mathrm{keV}$ [5]. The first successful STEM correctors were limited by $5^{\text {th }}$ order 4 -fold astigmatism $\mathrm{C}_{5,4}$, illustrated by the teal curves in Fig. 1. Next came the correction of all $5^{\text {th }}$ order aberrations and lower-order parasitic aberrations [6]. The probe size then became limited by chromatic aberration (red curves), with a stronger dependence on the primary energy than geometric aberrations, making the $\mathrm{C}_{\mathrm{c}}$ limit especially severe at lower keVs.

Overcoming the chromatic aberration limit can be achieved by eliminating $\mathrm{C}_{\mathrm{c}}$ with a chromatic aberration corrector, or by reducing the energy spread $\delta \mathrm{E}$ substantially with a monochromator. The second option was tried in 2012 [7], without convincing results. We have chosen this option, which reduces the available beam current, but has the major advantage that it leads to higher energy resolution in EELS, unlocking new experimental capabilities [4]. At $30 \mathrm{keV}$, the non-monochromated probe size in our microscope system is about $165 \mathrm{pm}$, and reducing $\delta \mathrm{E}$ from 350 to $100 \mathrm{meV}$ lowers this limit $1.8 \mathrm{x}$. Capturing the $107 \mathrm{pm}$ spacing in graphene then becomes possible at $30 \mathrm{kV}$, provided that geometric 
aberrations are controlled well enough for 50 mrad illumination half-angle. This performance is demonstrated by the graphene image and FFT in Fig. 2, and marked by the blue diamond in Fig. 1. The next fundamental limit for quadrupole-octupole correctors is then posed by 8 -fold astigmatism $\mathrm{C}_{7,8}$, indicated by the blue curves in Fig. 1.

Recording a $107 \mathrm{pm}$ spacing at $30 \mathrm{keV}(\lambda=6.98 \mathrm{pm})$ demonstrates spatial resolution of $15 \lambda$. This is, as far as we know, the best resolution performance for any transmission electron microscope. It has also been reached by a $\mathrm{C}_{\mathrm{c}}$-corrected fixed-beam TEM [7] and by a low $\mathrm{C}_{\mathrm{c}}$ STEM [8], but with lower contrast levels. Progressing further with the monochromation approach will become possible if brighter electron sources, which would also be very useful for monochromated EELS, are developed. If seventh-order aberrations are also overcome, STEM will become limited by $9^{\text {th }}$ order aberrations such as $\mathrm{C}_{9,10}$, whose influence is estimated by the green curves in Fig. 1. Spatial resolution may then progress to $\sim 7 \lambda$, which would make sub-100 pm resolution possible at $10 \mathrm{keV}$ primary energy, and $<30 \mathrm{pm}$ at $100 \mathrm{keV}$.

\section{References:}

[1] P.E. Batson, N. Dellby and O.L. Krivanek, Nature 418 (2002), p. 617.

[2] O.L. Krivanek et al, Nature 464 (2010), p. 571.

[3] O.L. Krivanek et al, in "Scanning Transmission Electron Microscopy" eds, Pennycook S.J. and Nellist P.D. (Springer) p. 613.

[4] Krivanek et al, Nature 514 (2014), p. 209.

[5] P.D. Nellist et al, Science 305 (2004), p. 1741.

[6] O.L. Krivanek et al, Ultramicroscopy 108 (2008), p. 179.

[7] R. Schillinger, http://www.emc2012.org.uk//documents/Abstracts/Abstracts/EMC2012_0940.pdf

[8] M. Linck et al, Phys. Rev. Letts 117 (2016), p. 076101.

[9] H. Sawada et al, Phys. Rev. Letts 114 (2015), p. 166102.

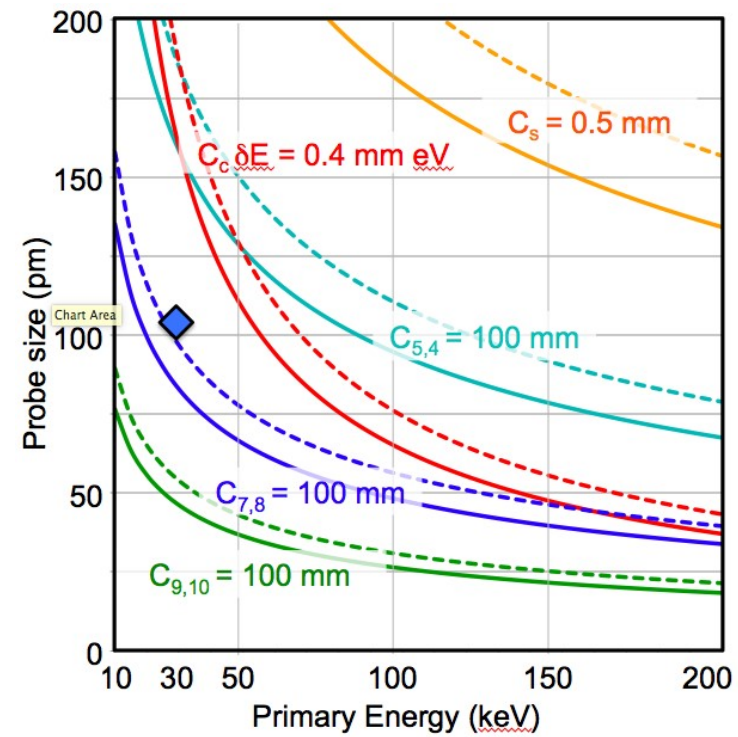

Figure 1. Theoretical STEM probe sizes for different types of limiting aberrations. Solid curves correspond to $\mathrm{I}_{\mathrm{p}} / \mathrm{I}_{\mathrm{c}}=0.1$, dashed curves to $\mathrm{I}_{\mathrm{p}} / \mathrm{I}_{\mathrm{c}}=0.5$. The blue diamond shows the performance demonstrated in Fig. 2.

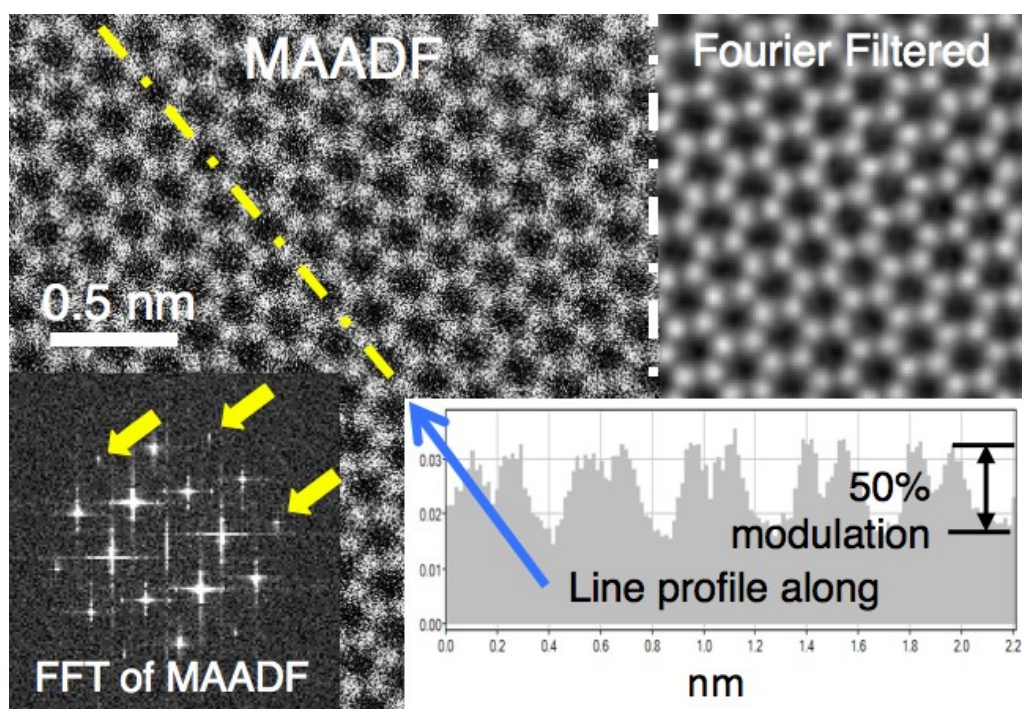

Figure 2. Medium-angle annular dark field (MAADF) STEM image of graphene obtained with Nion U-HERMES ${ }^{\mathrm{TM}} 100$ at 30 $\mathrm{keV}$, with FFT and a line profile taken along the indicated direction. $\mathrm{C}_{3}$ and $\mathrm{C}_{5}$-corrected, energy spread $\delta \mathrm{E} \sim 100 \mathrm{meV}$, $\mathrm{C}_{\mathrm{c}}=0.96 \mathrm{~mm}$. Arrows in the FFT point to strongly transferred [2020] 107 pm graphene spacings. 